\title{
Dexamethasone Intravitreal Implant in Diabetic Macular Edema: Real-Life Data from a Prospective Study and Predictive Factors for Visual Outcome
}

\author{
Irini Chatziralli (D) Panagiotis Theodossiadis · Efstratios Parikakis · \\ Eleni Dimitriou • Tina Xirou · George Theodossiadis · Stamatina A. Kabanarou
}

Received: October 8, 2017 / Published online: November 6, 2017

(C) The Author(s) 2017. This article is an open access publication

\begin{abstract}
Introduction: The purpose of the study was to evaluate the long-term anatomical and functional outcomes in patients with diabetic macular edema (DME) treated with intravitreal dexamethasone implant and to determine the predictive factors for the final visual outcome.

Methods: The study included 54 patients with DME refractory to previous antivascular endothelial growth factor (anti-VEGF) who were treated with intravitreal dexamethasone implant. Predictive factors for visual outcome were assessed. In addition, the change in best-corrected visual acuity (BCVA) and the percentage of patients with edema resolution were evaluated.
\end{abstract}

Enhanced Content To view enhanced content for this article go to http://www.medengine.com/Redeem/ BDCCF060316E7CB0.

I. Chatziralli $(\bowtie) \cdot$ P. Theodossiadis · E. Dimitriou G. Theodossiadis

2nd Department of Ophthalmology, Attikon

Hospital, National and Kapodistrian University of

Athens, Athens, Greece

e-mail: eirchat@yahoo.gr

E. Parikakis

2nd Department of Ophthalmology,

Ophthalmiatrion Athinon, Athens, Greece

T. Xirou $\cdot$ S. A. Kabanarou

Retina Department, Red Cross

"Korgialeneio-Benakeio" Hospital, Athens, Greece
Results: At the end of the 12-month follow-up, patients with DME gained +5.2 letters (about 1 Snellen line), while $57.4 \%$ of patients presented total resolution of macular edema. Negative predictive factors for the final visual outcome were found to be increasing age, increasing macular thickness, phakic status, the presence of intraretinal fluid, hyperreflective foci, hard exudates, as well as external limiting membrane and ellipsoid zone disruption. The mean number of injections was 2.1.

Conclusions: The various predictive factors that determine the visual outcome and possibly define patient prognosis after dexamethasone intravitreal implant in DME cases have been studied. The long follow-up showed that dexamethasone intravitreal implant seems to be a safe and effective treatment for this disease, requiring a limited number of injections.

Keywords: Diabetic macular edema; Dexamethasone; Optical coherence tomography; Predictive factors; Visual acuity

\section{INTRODUCTION}

Diabetic retinopathy (DR) is the leading cause of visual impairment in working-age populations in the developed world [1], while diabetic macular edema (DME) is the most common cause of vision loss in patients with DR, affecting about $20 \%$ of patients with DR [2]. DME 
results from blood-retina barrier disruption as a response to chronic hyperglycemia, leading to vascular leakage, fluid accumulation, and macular thickening [3]. Furthermore, inflammation seems to be implicated in the pathophysiology of DME, since several cytokines and chemokines, such as vascular endothelial growth factor (VEGF), tumor necrosis factor- $\alpha$ (TNF- $\alpha$ ), intercellular adhesion molecule-1 (ICAM-1), interleukin-6 (IL-6), and monocyte chemotactic protein-1 (MCP-1), have been found to be overexpressed in DME, increasing vascular permeability and leukostasis and altering fluid homeostasis within the neuroretinal tissue [4-6].

For many years, standard care for DME was focal/grid laser photocoagulation along with medical control of diabetes mellitus, blood pressure control, and lipid management [7]. Nowadays, the treatment of choice in DME is intravitreal anti-VEGF agents, which have been proven to be safe and effective at improving visual acuity (VA) and reducing macular thickness in patients with DME in large clinical trials [8-11]. However, the need for frequent injections and the fact that some patients do not respond to anti-VEGF agents mean that there is an unmet need for additional treatment alternatives for patients with DME $[9,12]$. Since inflammation plays a significant role in the pathogenesis of DME, intravitreal steroids have been shown to be useful in the treatment of DME, as they inhibit inflammation, leukostasis, and phosphorylation of cell-junction proteins, and they block the production of VEGF and other inflammatory mediators in DME $[13,14]$.

Dexamethasone intravitreal implant (Ozurdex; Allergan Inc., Irvine, CA, USA) is a biodegradable, sustained-release implant that has been approved for the treatment of DME based on a large phase III prospective study, the MEAD study, which showed a significant improvement in VA and macular edema after 3 years of follow-up in patients treated with $0.7 \mathrm{mg}$ dexamethasone intravitreal implant compared to a sham group [12]. However, real-world patients are generally less healthy and have potentially confounding comorbidities, and they are not selected based on strict eligibility criteria, unlike patients in clinical trials [15]. Therefore, observational studies of the use of a treatment in clinical practice provide useful information regarding patterns of use and the effectiveness of the treatment in a real-life setting [15]. While studies have been conducted to assess the use of dexamethasone intravitreal implant for the treatment of DME, they have mainly been retrospective, with limited follow-up [16-21].

In light of the above, the purpose of this prospective study was to evaluate the effectiveness and safety of dexamethasone intravitreal implant in patients with DME in real-life clinical practice with a long-term follow-up, and to investigate the potential predictive factors of the final visual outcome using multivariate analysis.

\section{METHODS}

Participants in this prospective study were 54 patients (54 eyes) with DME refractory to previous treatment with anti-VEGF agents who were treated with dexamethasone intravitreal implant at three retina departments in Greece between March 2015 and June 2016 and had at least 12 months of follow-up. All procedures followed were in accordance with the ethical standards of the responsible committee on human experimentation (institutional and national) and with the Helsinki Declaration of 1964, as revised in 2013. Informed consent was obtained from all patients before they were included in the study.

All patients had DME (central subfield thickness, CST $>320 \mu \mathrm{m}$ ) and had previously been treated with at least three intravitreal anti-VEGF injections to the affected eye, which then showed no response, defined as no increase in VA and no reduction in CST. Previous anti-VEGF injections were administered on a pro re nata (PRN) basis, according to specific retreatment criteria, including loss of VA of more than one Snellen line, increase in CST of $>50 \mu \mathrm{m}$, and presence of intraretinal (IRF)/subretinal (SRF) fluid compared to the previous visit. Patients with macular edema secondary to a cause other than diabetes mellitus, macular ischemia, a history of vitrectomy, intraocular 
surgery in the previous 6 months, a history of systemic corticosteroids within 6 months before baseline evaluation, uveitis, glaucoma or ocular hypertension, dense cataract, and those lost to follow-up were excluded from the study.

All participants received dexamethasone intravitreal implant at baseline under a sterile protocol that included the use of $5 \%$ povidone-iodine solution, topical anesthesia, eyelid-speculum application, intravitreal injection of $0.7 \mathrm{mg}$ dexamethasone implant via pars plana in the inferotemporal quadrant at $4 \mathrm{~mm}$ from the limbus in phakic eyes and $3.5 \mathrm{~mm}$ in pseudophakic eyes, followed by postoperative topical antibiotic eye drops. Re-injection was performed 6 months after the first injection if the patient experienced decreased vision and/or an increase in CST secondary to recurrent/worsening of DME, and at the clinician's discretion.

We recorded demographic data, such as age, gender, duration of diabetes mellitus, duration of DME, HbA1c levels, DR status, previous treatment, and other comorbidities. We also documented the clinic-based best-corrected VA (BCVA) measured by Snellen charts, the CST on spectral domain optical coherence tomography (SD-OCT; Spectralis HRA-OCT, Heidelberg Engineering, Heidelberg, Germany), the presence of IRF, SRF, hyperreflective foci and hard exudates, the status of the ellipsoid zone and the external limiting membrane (ELM), the presence of vitreomacular traction (VMT) and an epiretinal membrane (ERM), as well as intraocular pressure (IOP) at every monthly visit. OCT scans were analyzed by two graders who were not blinded to clinical data (IC, SK), and the interobserver agreement was almost perfect $(k>0.80)$. At baseline, a fluorescein angiogram (FA; Spectralis HRA-OCT, Heidelberg Engineering) was also obtained to confirm the diagnosis and evaluate macular ischemia.

Efficacy was measured using the mean changes in BCVA and CST from the baseline, as well as by calculating the percentages of patients gaining $\geq 5$ letters, $\geq 10$ letters and $\geq 15$ letters in VA and the percentage of those with edema resolution at the end of the 12month follow-up. Safety was assessed by monitoring changes in IOP, the use of IOP-lowering agents, the incidence rates of glaucoma and cataract surgery, and investigator-reported adverse events. In addition, predictive factors for final visual outcome were evaluated, such as age, gender, the duration of diabetes mellitus, the duration of macular edema, HbA1c level, the status of DR (nonproliferative or proliferative), the presence of hypertension and hyperlipidemia, any previous treatment, CST, the presence of SRF, IRF, hyperreflective foci, and hard exudates, the status of the ellipsoid zone and the ELM, and the presence of VMT and an ERM.

\section{Statistical Analysis}

To describe patient characteristics at baseline, mean \pm standard deviation (SD) values were used for continuous variables and counts with percentages for categorical variables. For longitudinal comparisons of BCVA and CST between the baseline and each time point, the Wilcoxon matched-pairs signed-rank test was used; given that ten comparisons were made, the level of statistical significance was set at $0.05 / 5=0.01$, according to the Bonferroni correction. BCVA was converted to ETDRS letters for statistical purposes.

To assess factors that may determine the VA, generalized least squares (GLS) random-effects linear regression analysis was performed (this is appropriate for longitudinal data given the intercorrelation of observations in such datasets). BCVA was the dependent variable. The aforementioned factors that were assessed as potential predictors for visual acuity were set as independent variables in models that were always adjusted for time (in months). The beta coefficients along with their 95\% confidence intervals (CIs) are provided in the manuscript.

Statistical analysis was performed using SPSS 22.0 (SPSS Inc, Chicago, IL, USA). A $p$ value $<0.05$ was considered to be statistically significant, except in cases where the Bonferroni correction was adopted, as declared above.

\section{RESULTS}

Table 1 shows the demographic and clinical characteristics of our study sample. 54 patients 
Table 1 Demographic and clinical characteristics of our study sample at baseline

\begin{tabular}{ll}
\hline & $\begin{array}{l}\text { Patients with diabetic macular } \\
\text { edema }(\boldsymbol{n} \mathbf{5} \mathbf{5})\end{array}$ \\
& Mean \pm standard deviation \\
\hline Age (years) & $69.2 \pm 7.6$ \\
$\begin{array}{l}\text { Duration of diabetes } \\
\text { mellitus (years) }\end{array}$ & $15.9 \pm 7.1$ \\
HbAlc (\%) & $7.2 \pm 0.6$ \\
Best-corrected visual & $52.0 \pm 13.4$ \\
acuity (letters) & \\
Best-corrected visual & $20 / 80$ \\
acuity (Snellen) & \\
Central retinal & $537.6 \pm 174.9$ \\
thickness ( $\mu$ m) & $13.3 \pm 1.4$ \\
Intraocular pressure \\
(mmHg)
\end{tabular}

with DME were included in the study. The mean age of patients was $69.2 \pm 7.6$ years. 35 patients $(64.8 \%)$ were male and $19(35.2 \%)$ were female. The mean duration of diabetes mellitus was $15.9 \pm 7.1$ years, but all patients were relatively well controlled with a mean $\mathrm{HbA1c}$ of $7.2 \pm 0.6 \% .46 .3 \%$ of the patients had suffered from DME for less than 6 months, while $53.7 \%$ had DME for 6 months or more. Regarding comorbidities, $88.9 \%$ and $44.4 \%$ of the patients had hypertension and hyperlipidemia, respectively. At baseline, 48 patients $(88.9 \%)$ had nonproliferative DR and $11.1 \%$ had proliferative DR that was previously treated with panretinal photocoagulation. All patients were previously treated for DME with anti-VEGF agents, and some of them received additional macular laser photocoagulation. Specifically, $85.2 \%$ of the patients had received previous intravitreal ranibizumab injections and 13\% intravitreal aflibercept injections, while $13 \%$ of the patients had additional focal/grid laser photocoagulation. $74.1 \%$ of the patients were pseudophakic, while $25.9 \%$ were phakic.

At baseline, the mean BCVA was $52.0 \pm 13.4$ ETDRS letters (20/80 Snellen). Figure 1 shows the evolution of BCVA over time; it shows that there was a statistically significant improvement in BCVA between all time points and the baseline $(p<0.001)$. At the end of the 12 -month follow-up period, patients with DME who were treated with dexamethasone intravitreal implant gained +5.2 letters (about one Snellen line). At month 12, 29 out of 54 patients $(53.7 \%)$ showed an improvement in BCVA. Specifically, $53.7 \%$ gained $\geq 5$ letters, $29.6 \%$ gained $\geq 10$ letters, and $14.8 \%$ gained $\geq 15$ letters. $33.3 \%$ of the patients remained stable. On the other hand, about $13.0 \%$ of the patients lost vision at the end of the follow-up, as shown in Fig. 2.

At baseline, the mean CST was $537.6 \pm 174.9 \mu \mathrm{m}$. Figure 3 shows the evolution of CST over time, illustrating that there was a statistically significant reduction in CST at all time points compared to the baseline $(p<0.001$ for all comparisons). At month 12, the CST was significantly decreased by $181 \mu \mathrm{m}$, while the maximum decrease in CST was observed at month $1(-198 \mu \mathrm{m})$. At the end of the 


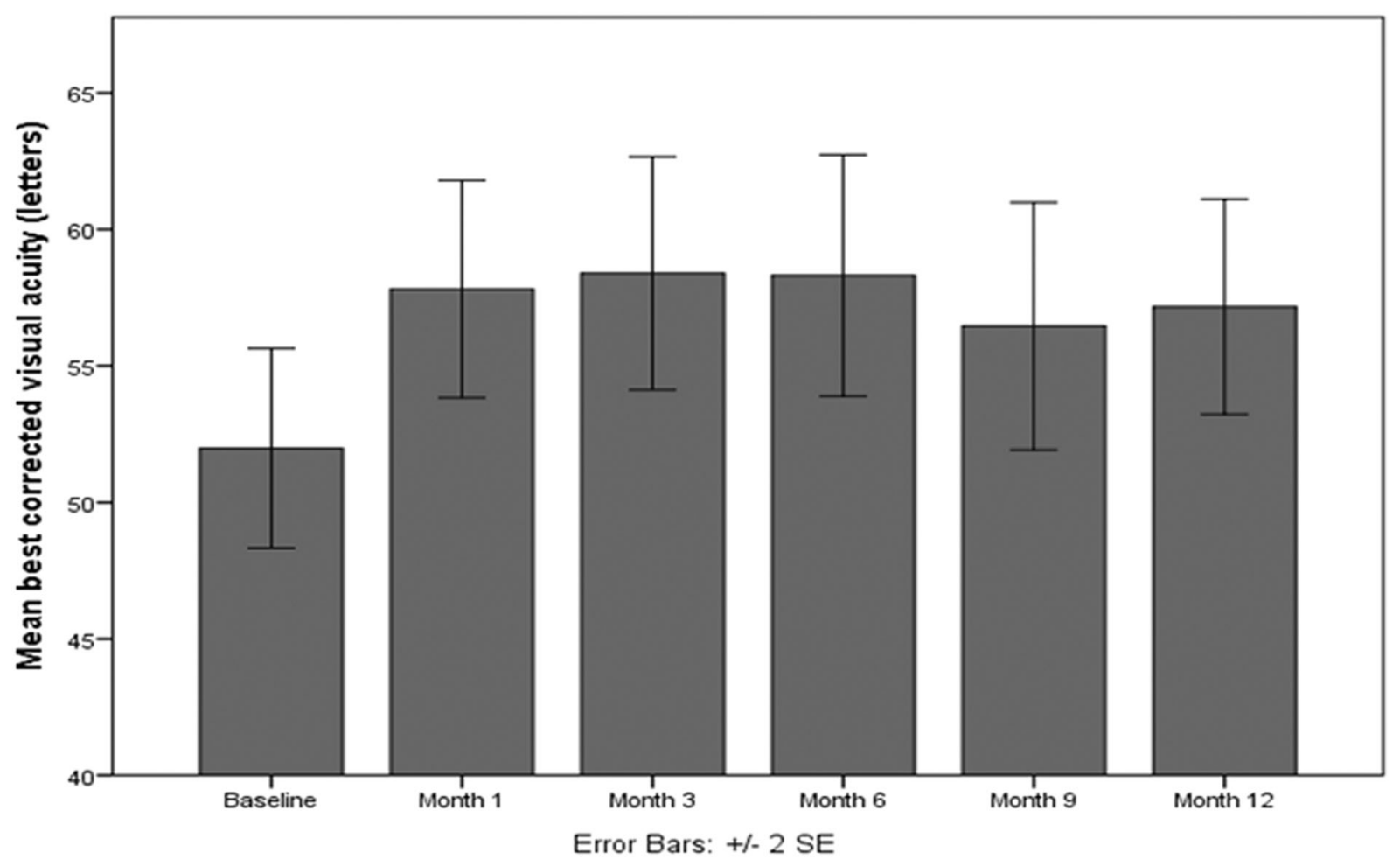

Fig. 1 Evolution of visual acuity in patients with diabetic macular edema over time

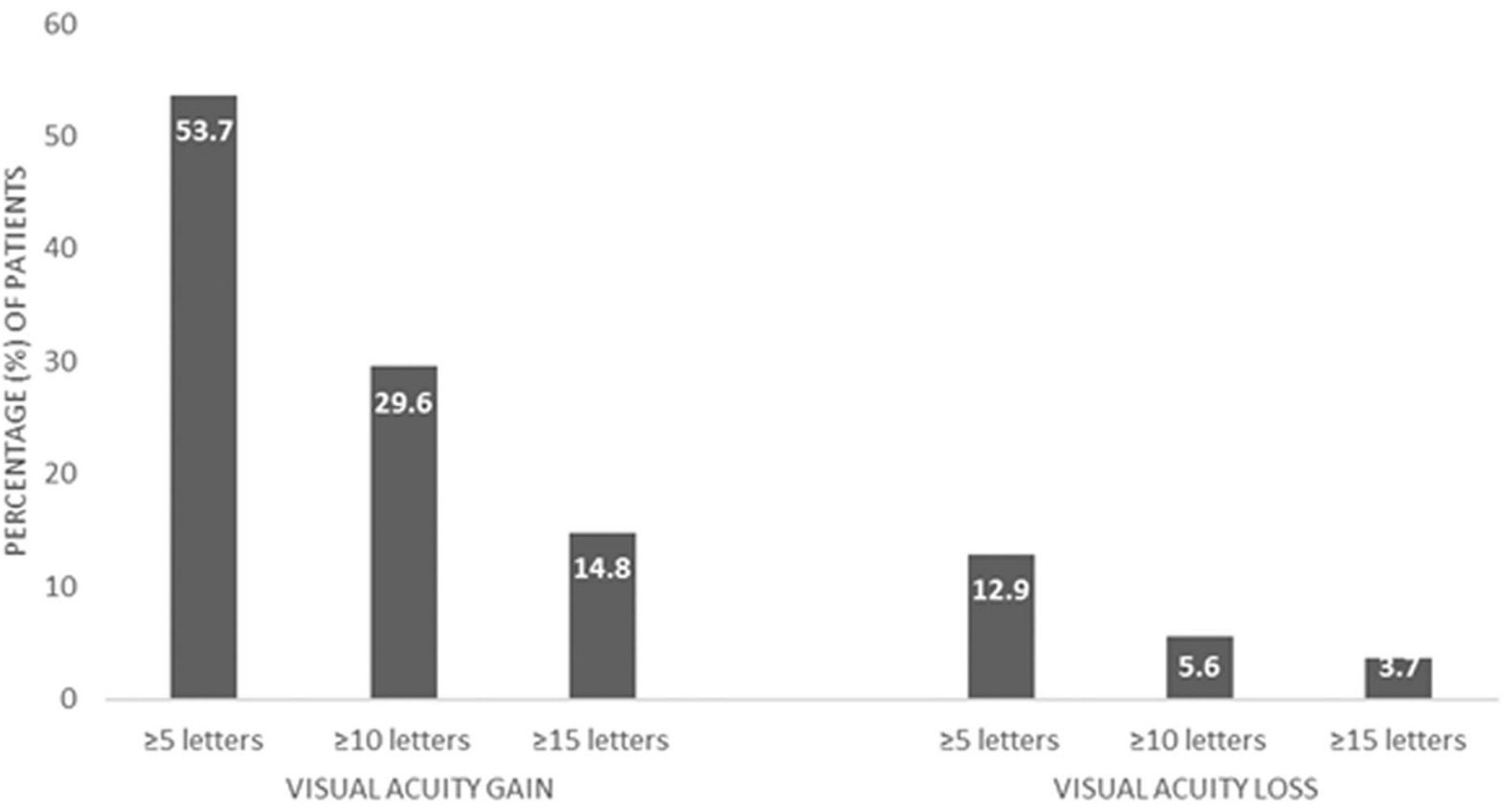

Fig. 2 Percentages of the patients who had gained or lost $\geq 5, \geq 10$, and $\geq 15$ letters at the 12-month follow-up 


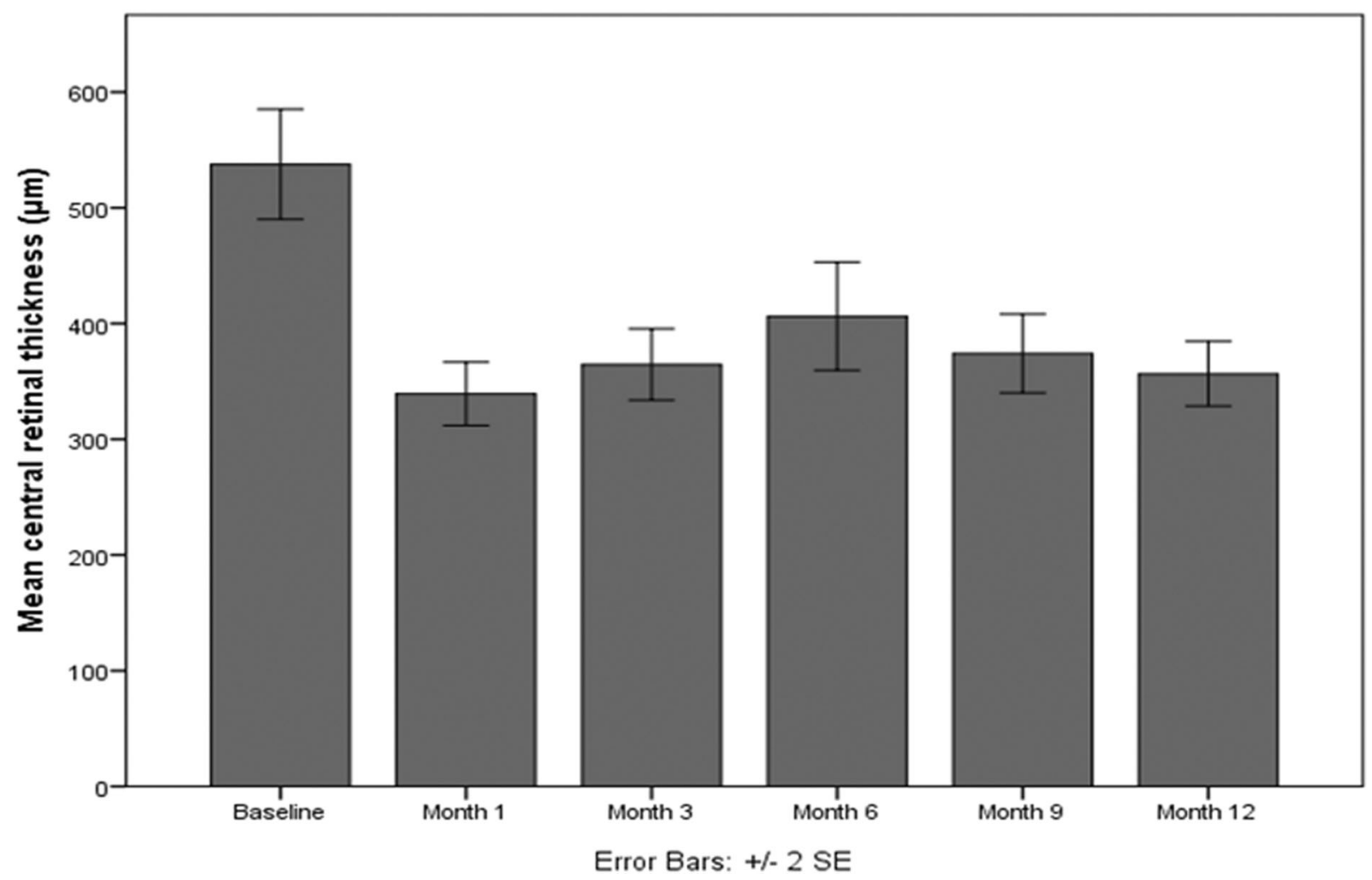

Fig. 3 Evolution of central subfield thickness in patients with diabetic macular edema over time

12-month follow-up, 31 patients (57.4\%) presented total resolution of macular edema (no IRF/no SRF).

At baseline, none of the patients had macular ischemia, as these patients were excluded from the study. The mean number of injections was $2.1 \pm 0.6$ at the end of the follow-up.

The results of the GLS linear regression analysis tha examined the factors associated with final visual acuity (letters) are presented in Table 2. Increasing age (coefficient $=-2.78$, $95 \% \mathrm{CI}=-5.21$ to $-1.13, p<0.001)$, increasing CST (coefficient $=-5.23,95 \% \mathrm{CI}=-7.93$ to $-3.56, p<0.001$ ), phakic status (coefficient $=-2.05, \quad 95 \% \quad \mathrm{CI}=-4.53$ to +0.15 , $p=0.043$ ), presence of IRF (coefficient $=-3.46$, $95 \% \mathrm{CI}=-5.63$ to $-1.81, p \leq 0.001$ ), hyperreflective foci (coefficient $=-6.02, \quad 95 \%$ $\mathrm{CI}=-10.12$ to $-2.21, p<0.001)$, hard exudates (coefficient $=-6.23,95 \% \mathrm{CI}=-9.13$ to $-2.82, p<0.001)$, ellipsoid zone disruption (coefficient $=-3.15,95 \% \mathrm{CI}=-4.73$ to -1.93 , $p \leq 0.001)$ and ELM disruption (coefficient $=-4.18,95 \% \mathrm{CI}=-6.24$ to -2.73 , $p \leq 0.001$ ) were all significantly associated with poorer VA. Final visual acuity was not associated with gender, hypertension, hyperlipidemia, DR status, duration of diabetes mellitus, duration of macular edema, HbA1c levels, or the presence of SRF, VMT, or an ERM.

As far as the complications are concerned, no serious systemic side effects were reported from any of the patients in the study. No thromboembolic or cardiovascular events were mentioned. In addition, there was no inflammatory reaction, endophthalmitis, or retinal tears. One patient $(1.9 \%)$ had retinal detachment and underwent pars plana vitrectomy with a good visual outcome. Two out of 14 phakic patients $(14.3 \%)$ developed cataract within the 12-month follow-up period and were scheduled for cataract surgery.

At baseline, IOP was $13.3 \pm 1.4 \mathrm{mmHg}$; it increased slightly at month 1 , although the change was not statistical significant $(15.2 \pm 2.3, p=0.047)$. The increase in IOP was 
Table 2 Results of the generalized least squares linear regression analysis that examined the factors associated with visual acuity (ETDRS letters). All models are adjusted for time (months)

\begin{tabular}{|c|c|c|c|}
\hline Variable & Category/increment & Coefficient $(95 \% \mathrm{CI})$ & $p$ value \\
\hline Age & Increase of 10 years & $-2.78(-5.21$ to -1.13$)$ & $<0.001$ \\
\hline Gender & Male vs female & $+1.15(-0.83$ to +2.19$)$ & 0.368 \\
\hline Hypertension & Yes vs no & $-2.11(-4.07$ to +1.01$)$ & 0.454 \\
\hline Hyperlipidemia & Yes vs no & $-3.32(-6.48$ to +1.54$)$ & 0.109 \\
\hline Lens status & Phakic vs pseudophakic & $-2.05(-4.53$ to +0.15$)$ & 0.043 \\
\hline Diabetic retinopathy status & Nonproliferative vs proliferative & $+2.09(+0.88$ to +5.12$)$ & 0.311 \\
\hline Duration of diabetes mellitus & 5 years increase & $-3.18(-5.51$ to +0.75$)$ & 0.289 \\
\hline Duration of macular edema & $\geq 6$ months vs $<6$ months & $-4.45(-6.09$ to -2.92$)$ & 0.083 \\
\hline HbAlc & $1 \%$ increase & $-3.39(-4.18$ to -2.83$)$ & 0.097 \\
\hline Central subfield thickness & $100 \mu \mathrm{m}$ increase & $-5.23(-7.93$ to -3.56$)$ & $<0.001$ \\
\hline Subretinal fluid & Yes vs no & $-1.73(-2.81$ to -0.65$)$ & 0.078 \\
\hline Intraretinal fluid & Yes vs no & $-3.46(-5.63$ to -1.81$)$ & $<0.001$ \\
\hline Hyperreflective foci & Yes vs no & $-6.02(-10.12$ to -2.21$)$ & $<0.001$ \\
\hline Hard exudates & Yes vs no & $-6.23(-9.13$ to -2.82$)$ & $<0.001$ \\
\hline Ellipsoid zone disruption & Yes vs no & $-3.15(-4.73$ to -1.93$)$ & $<0.001$ \\
\hline External limiting membrane disruption & Yes vs no & $-4.18(-6.24$ to -2.73$)$ & $<0.001$ \\
\hline Vitreomacular traction & Yes vs no & $-1.03(-2.27$ to -0.65$)$ & 0.882 \\
\hline Epiretinal membrane & Yes vs no & $-1.36(-2.14$ to -0.09$)$ & 0.531 \\
\hline
\end{tabular}

$p$ values shown in boldface are $<0.05$ and therefore considered to indicate statistical significance

transient and followed by a progressive decrease with no antihypertensive ocular treatment. Figure 4 shows the evolution of IOP during the follow-up. There was no statistically significant increase in IOP at month 12 compared to baseline. Only 3 patients $(5.6 \%)$ presented IOP $\geq 21 \mathrm{mmHg}$ at month 1 and received IOP-lowering drops. None of the patients underwent glaucoma surgery to reduce the IOP after injection. Figure 5 shows the evolution of one particular patient over time.

\section{DISCUSSION}

The principal conclusion of this study is that dexamethasone intravitreal implant appeared to be safe and effective for the treatment of
DME based on a relatively long follow-up period of 12 months in real-life clinical practice. Specifically, there was a mean improvement in BCVA of 5.2 letters at the end of the follow-up, while $87 \%$ of the patients presented an improvement in or a stabilization of VA. Total resolution of macular edema was observed in $57.4 \%$ of the patients at month 12 with a mean number of 2.1 injections. Furthermore, negative predictive factors for the final visual outcome in patients with DME that was treated with dexamethasone intravitreal implant were found to be increasing age, increasing CST, phakic status, presence of IRF, hyperreflective foci, hard exudates, and the disruption of the ellipsoid zone and ELM.

Our anatomical and functional results are in agreement with those of previous studies 


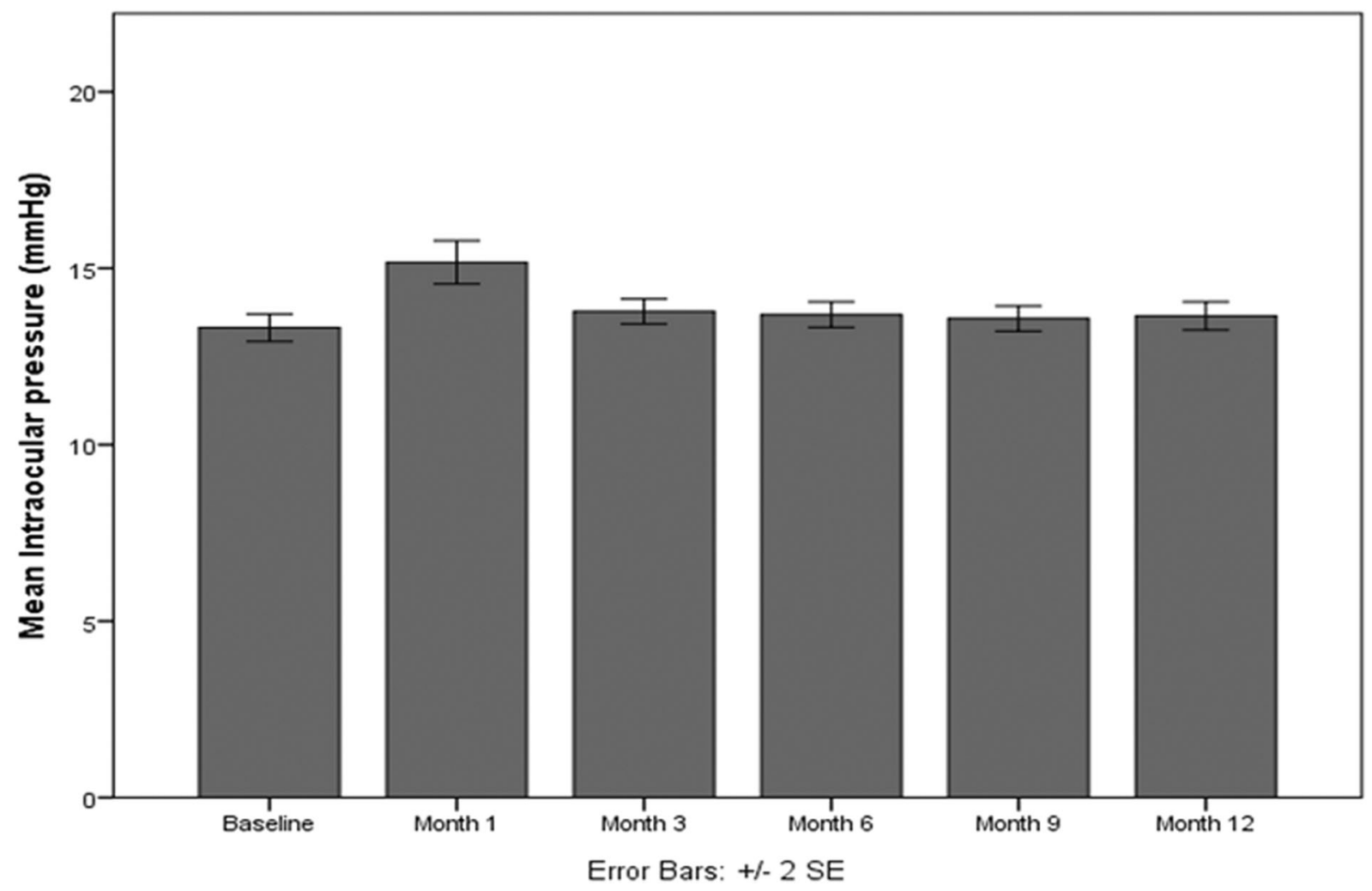

Fig. 4 Evolution over time of intraocular pressure in patients with diabetic macular edema that was treated with intravitreal dexamethasone implant

evaluating the efficacy of dexamethasone intravitreal implant in patients with DME based on real-life data, which noted a sustained BCVA and CST improvement without serious side effects [16-24]. It is worth noting that the peak efficacy of dexamethasone intravitreal implant, which has been shown to occur between the first and third months, correlates well with the concentration of the drug, which peaks in the vitreous at 2 months. The efficacy tends to slowly decrease from month 4 to month 6 , when CST usually increases [16].

In our study, a significant improvement in VA occurred in the first month and VA peaked at month 3 after one injection, while CST was markedly reduced at month 1 but gradually increased until month 6, when another injection was performed. This observation suggests that anatomically favorable results precede the functional outcomes, which can probably be attributed to the time needed to restore the photoreceptors. An interesting finding was that although the curves for BCVA and CST seemed to be symmetric, there was no significant correlation between them. Therefore, CST alone cannot predict the visual prognosis, while intraretinal alterations may explain this dissociation. Specifically, we found that the presence of IRF, hyperreflective foci, and hard exudates along with increasing CST was associated with unfavorable visual results. We hypothesize that morphological changes at the microstructural level from chronic and recurrent edema may lead to irreversible damage to photoreceptors, which is consistent with the fact that disruption of the ellipsoid zone and ELM was associated with poor visual acuity.

Furthermore, phakic lens status was found to be associated with a poorer VA. Previous studies found that the improvement in VA in patients treated with dexamethasone intravitreal implant was more prominent in pseudophakic eyes, as there was no effect of the lens [24]. Therefore, the effectiveness of the 

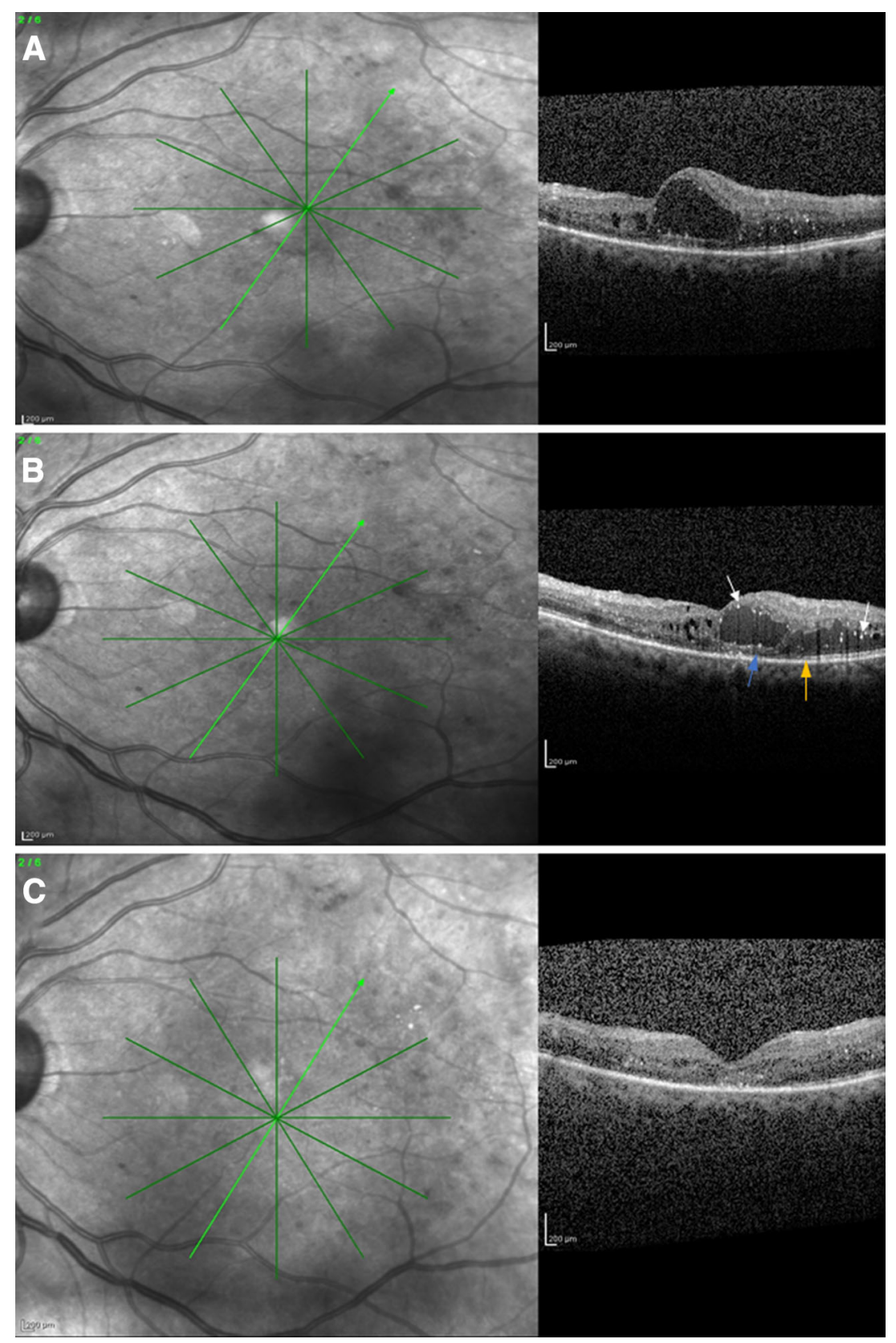

Fig. 5 a Spectral domain optical coherence tomography of a 54-year-old male patient who presented with diabetic macular edema and a visual acuity of 6/36 Snellen. b Spectral domain optical coherence tomography of the same patient after six ranibizumab injections, showing persistence of macular edema. The visual acuity was $6 / 36$ Snellen. Note the hyperreflective foci (white arrows), the

dexamethasone implant with respect to VA was more reliably appreciated by pseudophakic patients with DME. ellipsoid zone (orange arrow), and the external limiting membrane (blue arrow) disruption. c Spectral domain optical coherence tomography of the same patient after two intravitreal dexamethasone implant injections (month 12), where absorption of macular edema and decreased hyperreflective foci are apparent. The visual acuity was $6 / 9$ Snellen

Increasing age was also found to be a negative predictor for the final visual outcome in patients with RVO in our study, potentially due 
to age-related structural changes in the vessels of patients [25], but further investigation is needed to explain this phenomenon. On the other hand, it is worth mentioning that there was no association between the final visual outcome and the systemic factors (hypertension, hyperlipidemia, DR status, HbA1c levels) in our study. Thus, controlling these factors may not affect the final visual acuity, but it may protect from DR and DME progression, as was observed in a recent study by Guigou et al. [22]. It is worth noting that in our study, the patients were older than in the MEAD study. Moreover, although the patients in our study were better controlled than those in the MEAD study (who had similar durations of diabetes), with a mean HbA1c of $7.2 \pm 0.6 \%$ in this study compared to $7.6 \pm 1.2 \%$ in the MEAD study, there were also proliferative DR cases in our study.

Corticosteroids have some side effects, such as cataract development and increased IOP. In our study, only $14.3 \%$ of the phakic patients presented a cataract at the end of the follow-up period, but it should be noted that cataracts may also progress in patients with diabetes mellitus due to the disease per se. A rise in IOP was observed mainly at month 1 but it was moderate, transient, and followed by a progressive decrease. Only $5.6 \%$ of the patients presented $\mathrm{IOP} \geq 21 \mathrm{mmHg}$ at month 1 and received IOP-lowering drops, while no glaucoma surgery was needed to control the IOP. Another adverse event in our series was retinal detachment in one patient $(1.9 \%)$. This percentage is slightly higher than that for the MEAD study (0.3\%), but it should be noted that this patient had myopia, which could be an additional factor in retinal detachment.

A potential limitation of our study is the lack of a control group, while treatment-naïve patients are not included in the study. Moreover, the nature of GLS regression models inherently precluded the incorporation of the baseline BCVA as a separate covariate in the model; in this context, baseline values are simply predicted as linear combinations in the respective equations (the values at other time points are predicted in a similar manner), assuming linearity during the study period. However, the strengths of our study are the relatively large sample size of patients with macular edema due to DME and the long follow-up period of 12 months. Furthermore, as we used a spectral-domain OCT in our study, in contrast to the time-domain OCT used in the MEAD study (OCT 2 and 3), we were able to evaluate retinal structures in more detail before and after treatment, allowing us to identify predictive factors for visual outcomes.

In conclusion, dexamethasone intravitreal implant was found to be safe and effective for the treatment of patients with DME. A remarkable decrease in CST was observed at month 1 , followed by an improvement in VA, which also depended mainly on intraretinal changes (IRF, hyperreflective foci, hard exudates, condition of the ellipsoid zone and ELM). The study populations in randomized controlled trials are typically carefully selected using strict eligibility criteria. Therefore, the value of this type of observational study is that the effectiveness of treatment in real-life clinical practice is evaluated, leading to similar anatomical and functional results to those seen in previous randomized clinical trials. In addition, it is important to take the various predictive factors into account and inform each patient about their prognosis after treatment. Further studies with a control group and a large sample size are needed to validate our results and scrutinize the use of dexamethasone intravitreal implant in DME patients in everyday clinical practice.

\section{ACKNOWLEDGEMENTS}

No funding or sponsorship was received for the publication of this article.

All named authors meet the International Committee of Medical Journal Editors (ICMJE) criteria for authorship for this manuscript, take responsibility for the integrity of the work as a whole, and have given final approval to the version to be published.

Disclosures. Irini Chatziralli, Panagiotis Theodossiadis, Efstratios Parikakis, Eleni Dimitriou, Tina Xirou, George Theodossiadis, and 
Stamatina A. Kabanarou have nothing to disclose.

Compliance with Ethics Guidelines. All procedures followed were in accordance with the ethical standards of the responsible committee on human experimentation (institutional and national) and with the Helsinki Declaration of 1964, as revised in 2013. Informed consent was obtained from all patients before they were included in the study.

Data Availability. The datasets generated during and/or analyzed during the current study are available from the corresponding author on reasonable request.

Open Access. This article is distributed under the terms of the Creative Commons Attribution-NonCommercial 4.0 International License (http://creativecommons.org/licenses/ by-nc/4.0/), which permits any noncommercial use, distribution, and reproduction in any medium, provided you give appropriate credit to the original author(s) and the source, provide a link to the Creative Commons license, and indicate if changes were made.

\section{REFERENCES}

1. Wild S, Roglic G, Green A, Sicree R, King H. Global prevalence of diabetes: estimates for the year 2000 and projections for 2030. Diabetes Care. 2004;27:1047-53.

2. Yau JW, Rogers SL, Kawasaki R, et alma T, Klein BE, Klein R, Krishnaiah S, Mayurasakorn K, O'Hare JP, Orchard TJ, Porta M, Rema M, Roy MS, Sharma T, Shaw J, Taylor H, Tielsch JM, Varma R, Wang JJ, Wang N, West S, Xu L, Yasuda M, Zhang X, Mitchell $\mathrm{P}$, Wong TY, Meta-Analysis for Eye Disease (META-EYE) Study Group. Global prevalence and major risk factors of diabetic retinopathy. Diabetes Care. 2012;35:556-64.

3. Bhagat N, Grigorian RA, Tutela A, Zarbin MA. Diabetic macular edema: pathogenesis and treatment. Surv Ophthalmol. 2009;54:1-32.

4. Das A, McGuire PG, Rangasamy S. Diabetic macular edema: pathophysiology and novel therapeutic targets. Ophthalmology. 2015;122:1375-94.
5. Barouch FC, Miyamoto K, Allport JR, Fujita K, Bursell SE, Aiello LP, Luscinskas FW, Adamis AP. Integrin-mediated neutrophil adhesion and retinal leukostasis in diabetes. Invest Ophthalmol Vis Sci. 2000;41:1153-8.

6. Crosby-Nwaobi R, Chatziralli I, Sergentanis T, Dew T, Forbes A, Sivaprasad S. Cross talk between lipid metabolism and inflammatory markers in patients with diabetic retinopathy. J Diabetes Res. 2015;2015:191382.

7. American Diabetes Association. Standards of medical care in diabetes-2009. Diabetes Care. 2009;32(Suppl 1):S13-61.

8. Mitchell P, Bandello F, Schmidt-Erfurth U, Lang GE, Massin P, Schlingemann RO, Sutter F, Simader C, Burian G, Gerstner O, Weichselberger A, RESTORE Study Group. The RESTORE study: ranibizumab monotherapy or combined with laser versus laser monotherapy for diabetic macular edema. Ophthalmology. 2011;118:615-25.

9. Brown DM, Nguyen QD, Marcus DM, Boyer DS, Patel S, Feiner L, Schlottmann PG, Rundle AC, Zhang J, Rubio RG, Adamis AP, Ehrlich JS, Hopkins JJ, RIDE and RISE Research Group. Long-term outcomes of ranibizumab therapy for diabetic macular edema: the 36-month results from two phase III trials: RISE and RIDE. Ophthalmology. 2013;120:2013-22.

10. Elman MJ, Ayala A, Bressler NM, Browning D, Flaxel CJ, Glassman AR, Jampol LM, Stone TW, Diabetic Retinopathy Clinical Research Network. Intravitreal ranibizumab for diabetic macular edema with prompt versus deferred laser treatment: 5-year randomized trial results. Ophthalmology. 2015;122:375-81.

11. Brown DM, Schmidt-Erfurth U, Do DV, Holz FG, Boyer DS, Midena E, Heier JS, Terasaki H, Kaiser PK, Marcus DM, Nguyen QD, Jaffe GJ, Slakter JS, Simader C, Soo Y, Schmelter T, Yancopoulos GD, Stahl N, Vitti R, Berliner AJ, Zeitz O, Metzig C, Korobelnik JF. Intravitreal aflibercept for diabetic macular edema: 100-week results from the VISTA and VIVID studies. Ophthalmology. 2015;122:2044-52.

12. Boyer DS, Yoon YH, Belfort R Jr, Bandello F, Maturi RK, Augustin AJ, Li XY, Cui H, Hashad Y, Whitcup SM, MEAD Ozurdex Study Group. Three-year, randomized, sham-controlled trial of dexamethasone intravitreal implant in patients with diabetic macular edema. Ophthalmology. 2014;121:1904-14.

13. Wang K, Wang Y, Gao L, Li X, Li M, Guo J. Dexamethasone inhibits leukocyte accumulation and vascular permeability in retina of 
streptozotocin-induced diabetic rats via reducing vascular endothelial growth factor and intercellular adhesion molecule-1 expression. Biol Pharm Bull. 2008;31:1541-6.

14. Tamura H, Miyamoto K, Kiryu J, Miyahara S, Katsuta $\mathrm{H}$, Hirose F, Musashi K, Yoshimura N. Intravitreal injection of corticosteroid attenuates leukostasis and vascular leakage in experimental diabetic retina. Invest Ophthalmol Vis Sci. 2005;46:1440-4.

15. Eter N, Mohr A, Wachtlin J, Feltgen N, Shirlaw A, Leaback R, German Ozurdex in RVO Real World Study Group. Dexamethasone intravitreal implant in retinal vein occlusion: real-life data from a prospective, multicenter clinical trial. Graefes Arch Clin Exp Ophthalmol. 2017;255:77-87.

16. Zhioua I, Semoun O, Lalloum F, Souied EH. Intravitreal dexamethasone implant in patients with ranibizumab persistent diabetic macular edema. Retina. 2015;35:1429-35.

17. Chhablani J, Bansal P, Veritti D, Sambhana S, Sarao V, Pichi F, Carrai P, Massaro D, Lembo A, Mansour AM, Banker A, Gupta SR, Hamam R, Lanzetta P. Dexamethasone implant in diabetic macular edema in real-life situations. Eye. 2016;30:426-30.

18. Ozkaya A, Alagoz C, Alagoz N, Gunes H, Yilmaz I, Perente I, Yazici AT, Taskapili M. Dexamethasone implant in pseudophakic and nonglaucomatous subgroup of diabetic macular edema patients: a real life experience. Eur J Ophthalmol. 2016;26:351-5.

19. Matonti F, Pommier S, Meyer F, Hajjar C, Merite PY, Parrat E, Rouhette H, Rebollo O, Guigou S. Long-term efficacy and safety of intravitreal dexamethasone implant for the treatment of diabetic macular edema. Eur J Ophthalmol. 2016;26:454-9.

20. Querques G, Darvizeh F, Querques L, Capuano V, Bandello F, Souied EH. Assessment of the real-life usage of intravitreal dexamethasone implant in the treatment of chronic diabetic macular edema in France. J Ocul Pharmacol Ther. 2016;32:383-9.

21. Malclès A, Dot C, Voirin N, Agard É, Vié AL, Bellocq D, Denis P, Kodjikian L. Real-life study in diabetic macular edema treated with dexamethasone implant: The Reldex Study. Retina. 2017;37:753-60.

22. Guigou S, Pommier S, Meyer F, Hajjar C, Merite PY, Parrat E, Rouhette H, Rebollo O, Matonti F. Efficacy and safety of intravitreal dexamethasone implant in patients with diabetic macular edema. Ophthalmologica. 2015;233:169-75.

23. Pacella F, Romano MR, Turchetti P, Tarquini G, Carnovale A, Mollicone A, Mastromatteo A, Pacella E. An eighteen-month follow-up study on the effects of intravitreal dexamethasone implant in diabetic macular edema refractory to anti-VEGF therapy. Int J Ophthalmol. 2016;9:1427-32.

24. Moon BG, Lee JY, Yu HG, Song JH, Park YH, Kim HW, Ji YS, Chang W, Lee JE, Oh J, Chung I. Efficacy and safety of a dexamethasone implant in patients with diabetic macular edema at tertiary centers in Korea. J Ophthalmol. 2016;2016:9810270.

25. Farinha C, Marques JP, Almeida E, Baltar A, Santos AR, Melo P, Costa M, Figueira J, Cachulo ML, Pires I, Silva R. Treatment of retinal vein occlusion with ranibizumab in clinical practice: longer-term results and predictive factors of functional outcome. Ophthalmic Res. 2015;55:10-8. 